\title{
Vigilancia tecnológica e inteligencia competitiva: conceptos, profesionales, servicios y fuentes de información
}

\author{
Por Elea Giménez Toledo y Adelaida Román Román
}

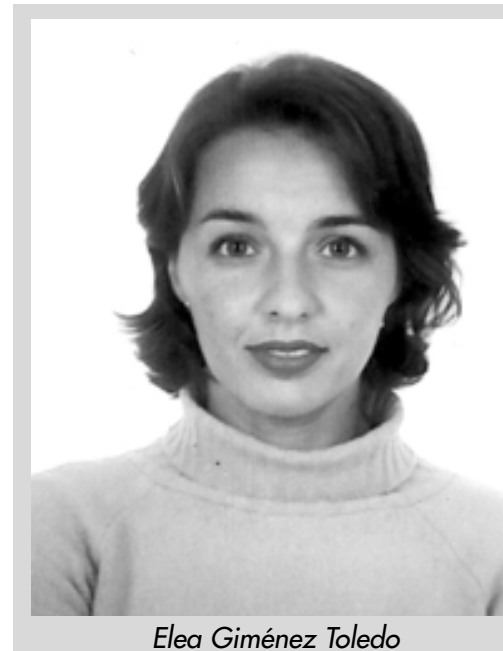

\begin{abstract}
Resumen: Las actividades de vigilancia tecnológica e inteligencia competitiva son herramientas de trabajo fundamentales para mejorar la competitividad de las organizaciones. Su éxito depende en gran medida de los sistemas de gestión del conocimiento en los que se apoyen. Este trabajo pretende dar a conocer la relación entre la gestión de la información y sus profesionales con las actividades de vigilancia tecnológica e inteligencia competitiva, así como presentar los principales agentes que trabajan en el área de la vigilancia tecnológica e inteligencia competitiva, fundamentalmente en España, y algunos portales de la escena internacional. Estas instituciones constituyen valiosas fuentes de información sobre la materia.
\end{abstract}

Palabras clave: Vigilancia tecnológica, Inteligencia competitiva, Profesionales de la información, Fuentes de información, Gestión del conocimiento.

Elea Giménez Toledo

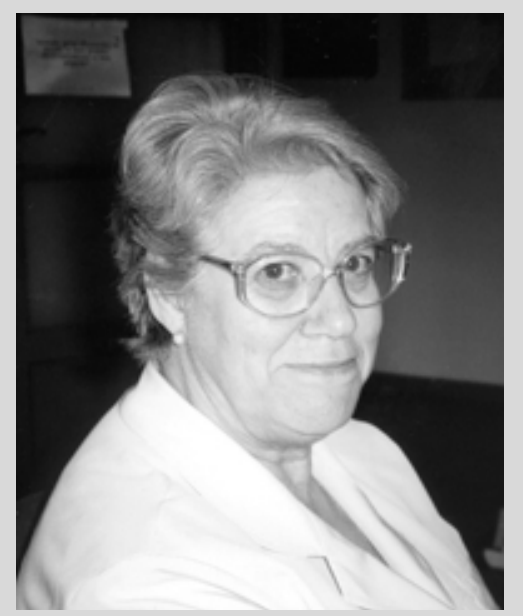

Adelaida Román Román

Title: Technology watch and competitive intelligence: concepts, professionals, services and information sources

Abstract: Technology watch and competitive intelligence activities form a basic tool for improving organizations' competitiveness. Their success depends on the knowledge management systems upon which they are based. This paper describes the relationship between information management (and professionals working in the field) and technology watch and competitive intelligence activities. Also presented are some of the major agents mainly in Spain - who are working in the area of technology watch and competitive intelligence, as well as some international portals. Together, these entities constitute a valuable source of information on this subject.

Keywords: Technology watch, Competitive intelligence, Information professionals, Information sources, Knowledge management.

Giménez Toledo, Elea; Román Román, Adelaida. "Vigilancia tecnológica e inteligencia competitiva: conceptos, profesionales, servicios y fuentes de información". En: El profesional de la información, 2001, mayo, v. 10, n. 5, pp. 11-20.

\section{Introducción}

La vigilancia tecnológica y la inteligencia económica o competitiva son procesos estrechamente unidos y orientados a la mejora de la competitividad de las empresas así como al aumento de la eficacia de las administraciones públicas, todo ello dentro un entorno innovador. Aunque en otros países como EUA, Canadá o Francia son prácticas implantadas desde hace tiempo, en España han empezado a cobrar fuerza sólo en los últimos años y ya se pueden encontrar empresas y organismos gestores de política científica que han incorporado paulatinamente este tipo de actividades a sus líneas de trabajo.

El objetivo de este análisis es presentar brevemente la relación de la información y sus profesionales con la vigilancia tecnológica así como dar a conocer algunas de las empresas e instituciones (sobre todo españolas) dedicadas a este ámbito y que se constituyen en fuentes de información sobre la materia pero también en ejemplos de cómo la gestión de la in- formación es la base de los sistemas de vigilancia tecnológica.

\section{La documentación y sus profesionales en la vigilancia tecnológica}

Aunque son muchas las definiciones que existen en torno a la vigilancia tecnológica y a la inteligencia competitiva creemos interesante destacar la que proponen Palop y Vicente $(1999$, b) sobre la primera de las expresiones: "sistema organizado de observación y análisis del entorno, tratamiento y circulación interna de los hechos observados y posterior utilización en la empresa". También es destacable el objetivo que se señala desde la Comunidad de Madrid para la vigilancia tecnológica, que es "la obtención continuada y el análisis sistemático de información de valor estratégico" (2000). La vigilancia tecnológica da paso o se incorpora dentro de la inteligencia económica, lo que supone utilizar la información obtenida relevante para la empresa de cara a la toma de decisiones acertada. Por esta razón se afirma que la vigilancia genera inteligencia. 


\section{Programa del Máster de inteligencia económica de la Ceram Sophia Antipolis}

\section{Traducción del programa disponible en:}

\section{http://www.ceram.edu/V2/}

- Herramientas del análisis estratégico (140 horas).

Inteligencia económica: un imperativo para la empresa.

De la información a la inteligencia.

De las redes informáticas a las redes humanas.

Conceptos relativos al tratamiento de la información elaborada.

Geopolítica: entorno económico, sociológico y cultural.

Organizaciones internacionales.

Estrategia geopolítica e inteligencia económica.

Los grandes mecanismos económicos y las claves para su lectura.

Aproximaciones culturales comparadas a los sistemas de otros países.

Ética y gestión.

Los nuevos retos de la estrategia empresarial.

De la estrategia de adaptación a la inteligencia estratégica: gestión de recursos.

Prospectiva y escenarios.

Gestión de las competencias.

Coacciones y dinámicas organizativas.

La estrategia de agentes.

Redes tecnológicas y transferencia de tecnología: obstáculos e intereses clave para evaluar $y$ entender las estrategias financieras internacionales.

El benchmarking.

Análisis y gestión de riesgos.

Gestión de la innovación.

Iniciación a las grandes familias tecnológicas.

La innovación: ventaja para el competidor, las oportunidades tecnológicas, dominio del proceso de innovación.

Inteligencia económica, vector de innovación.

Práctica de la comunicación y de la negociación para actuar como mediador en la empresa y fuera de ella.

Psicosociología de las organizaciones.

Dinámica de grupos.

Técnicas de negociación internacional.

De una situación extrema a la integración.

Comunicación y desarrollo personal.

- El mediador: un especialista en información elaborada. La información como herramienta (130 horas).

Metodología de la vigilancia tecnológica o la gestión de la incertidumbre.
Diferentes tipos de vigilancia tecnológica, competitiva, comercial y global.

Metodología para la información industrial. De la información a la acción: circulación inteligente de información; el observatorio del entorno y sus dispositivos específicos.

Elección de los sectores a vigilar: factores claves del éxito; información crítica.

Plan de información: estrategia y táctica de la recogida y la explotación de la información.

Gestión del proyecto "Inteligencia económica y vigilancia tecnológica": búsqueda, recogida, tratamiento, difusión; planificación y control de costes y control de su funcionamiento.

Informes entrada y salida: dossier general de información y dossier de información estratégica. Fichas "síntesis-acciones".

Fundamentos y teoría de la observación del entorno.

De la información abierta a la información cerrada.

La desinformación.

Condiciones generales de la información: legislación, distribución de la información, etc.

Tipos diferentes de información: de la información en bruto a la información elaborada.

Nuevas oportunidades: las autopistas de la información.

Estrategia de la propiedad industrial.

Oportunidades en relación con la propiedad industrial: aspectos jurídicos; las patentes.

Investigación de las formas de protección industrial: evaluación de la innovación, elección y costes de la protección, análisis de riesgos.

Minería de datos y cómo acceder a ellos.

Fuentes de información: naturaleza y estructura de las fuentes, minería de datos, evaluación y categorización de las fuentes de información, identificación de redes de expertos.

Presentación y principios para la interrogación de bases de datos (estructura, elección de bases de datos, lenguajes de interrogación, estrategias de búsqueda).

Localización de yacimientos de información.

Accesibilidad de la información.

Los problemas de la seguridad de la información.

Seguridad de las informaciones.

Seguridad informática. Sistemas informatizados y redes.
Protección de los conocimientos y del know-how de la empresa.

Organización de la salvaguardia de la información en la empresa

La seguridad en las redes: el caso de internet.

El tratamiento de la información y la realización de informes de inteligencia económica.

Introducción a la informática y a la ofimática.

Generalidades: contenido y formalización de un informe de inteligencia económica.

Programas informáticos de comunicación, estructura, parámetros y proceso de automatización de las demandas de información.

Herramientas para la realización de un informe de inteligencia económica.

Las autopistas de la información: estrategias de recopilación y de difusión.

Análisis de un informe: presentación, contenido, estructura.

Estudio de casos y trabajos prácticos.

- El mediador: un gestor de proyectos complejos, un especialista en gestión y organización. Herramientas de la gestión por proyectos (I30 horas).

Organización.

Organización y gestión de proyectos: la gestión de la complejidad, una cuestión de método.

Preparación, validación de proyectos complejos y viabilidad económica y financiera de los mismos.

Definición, preparación y gestión de los contratos, de los convenios con los socios, etc.

La gestión por proyectos en el sector público.

Implantación de un sistema de inteligencia económica en la empresa.

La función de mediador.

Presupuestos y costes de la inteligencia económica.

Creación de redes humanas internas y externas.

Coordinación de un grupo de trabajo integrado en un proyecto.

Implantación de un sistema básico de vigilancia para pymes/pymis.

Cultura de la información y cultura del lobby. Redes de influencia y de decisión a nivel micro y macroeconómico. 
Conocer el entorno, identificar tendencias (nuevos teAIMPLAS mas, nuevos actores, nuevos productos, nuevos procesos, etc.), riesgos, oportunidades, etc., y actuar en consecuencia, son los objetivos básicos de la vigilancia tecnológica y la inteligencia competitiva. El éxito de estos procesos depende de distintos factores pero podemos afirmar que hay dos determinantes. Por una parte, la participación de todos los miembros de una organización; por otra, la existencia de un sistema de gestión del conocimiento eficaz, entendiendo por tal un modelo de gestión de información formal e informal.

\section{«La vigilancia tecnológica y la inteligencia competitiva son pro- cesos estrechamente unidos y orientados a la mejora de la com- petitividad de las empresas»}

En la medida que el sistema de información es un pilar fundamental de las actividades de vigilancia tecnológica e inteligencia competitiva, el profesional de la información tiene un importante papel que desempeñar. Además su participación puede verse ampliada con la realización de estudios bibliométricos y

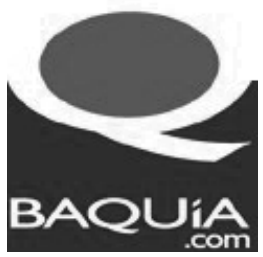
cienciométricos que permitan identificar áreas de investigación emergentes, tendencias de actuación de las empresas, etc., mediante el análisis de co-ocurrencias y la posterior elaboración de mapas tecnológicos.

Ambas actuaciones (gestión de la información y análisis bibliométrico) pueden ser importantes puntos de apoyo no sólo de la empresa (ámbito al que parece representar siempre la vigilancia tecnológica), sino también para las administraciones públicas, que empiezan a utilizar estas herramientas en la elaboración de sus planes de gobierno.

El papel del gestor de la información en los procesos de vigilancia tecnológica e inteligencia competitiva es aún muy limitado en las empresas españolas. En primer lugar, porque estas actividades no están ni lo suficientemente conocidas ni implantadas. Una CERAM SOPHIA ANTIPOLIS segunda razón es porque aún no se ha definido bien el perfil de un profesional de la vigilancia tecnológica e inteligencia competitiva. Sin embargo, basta con observar qué está ocurriendo fuera de nuestras fronteras para creer que el profesional de la información tiene un campo de acción en estos procesos: gestores del conocimiento que trabajan en vigilancia tecnológica, escuelas y facultades de biblioteconomía y documentación que imparten estudios y especialidades relacionados con el tema (por ejemplo: curso de inteligencia estratégica de la Escuela de Biblioteconomía y Documentación de la Universidad de Indiana, el curso "Vigilancia informacional estratégica" incluido en el Máster de ciencias de la información de la Universidad de Montreal, Máster de inteligencia competitiva de la Universidad de Aix-Marseille, Máster de inteligencia económica

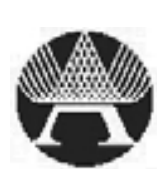

R E D ANDALUCIA INNUVACIÓN (c) del Ceram — véase programa adjunto-, etc.), utilización de herramientas típicas del trabajo documental (análisis bibliométricos, seguimientos y alertas informativas, etc.), y, sobre todo, la evidencia de que detrás de estos procesos siempre hay una recogida selectiva de información, un tratamiento y una difusión.

Sin embargo, es importante reconocer que la exploración de este territorio implica necesariamente la redefinición del perfil profesional del gestor de la información de forma que pueda integrarse en el ciclo completo de la vigilancia tecnológica. Esto supone añadir a los conocimientos técnicos propios de la profesión un profundo entendimiento de la empresa o institución para la que se trabaja, así como una comunicación eficaz con todos los demás implicados en vigilancia tecnológica.

\section{«La vigilancia genera inteligencia»}

Su aportación en esta tarea es importante, pero no única, y puede variar en función del grado de confianza o responsabilidad que se le otorgue desde la dirección. En este sentido conviene recordar los distintos perfiles profesionales que define la $A d b s$ centro de
vigilancia
normas y
patentes (Association des Profesionnels de l'Information et de la Documentation) en relación con las actividades de vigilancia tecnológica, que vienen dados, entre otras cosas, por la mayor o menor cercanía del trabajo del documentalista a la dirección de la institución. La labor de este profesional está asegurada en vigilancia tecnológica pero sólo participará en la generación de inteligencia en la medida en que pueda colaborar en el proceso de toma de decisiones.

Como el propio término indica, la vigilancia supone estar informado de lo que está ocurriendo en un determinado entorno. Para el gestor de la información esto significa detectar información de interés procedente de recursos variados y dispersos, pero también seguir y analizar aquellas otras fuentes de obligada consulta que habitualmente aportan información relevante y que deben explorarse sistemáticamente. Esta tarea se ve facilitada, cada vez en más ocasiones, por las alertas informativas que pueden generar los sistemas de 
información automatizados en torno a un perfil temático especificado por el usuario y que son ofrecidos por revistas científicas electrónicas, diarios de información general y especializada, boletines oficiales, etc. Además, las instituciones que desarrollan actividades de vigilancia tecnológica cuentan también con los servicios ofrecidos por empresas del sector documentación, en forma de selección de recursos de información especializados, estudios bibliométricos o, por ejemplo, el servicio spidering de DOC6 que permite analizar periódicamente algunas direcciones web que pueden ofrecer datos de interés para el cliente.

http://www.doc6.es

\section{«El papel del gestor de la infor- mación en los procesos de vigi- lancia tecnológica e inteligencia competitiva es aún muy limita- do en las empresas españolas»}

Este tipo de seguimiento informativo se enmarca dentro de la "vigilancia sobre internet", término que alude a la necesidad de las instituciones de rastrear sistemáticamente la Red en busca de información relevante y para lo cual recurre a todas las herramientas de recuperación útiles para su propósito. Sin embargo, la vigilancia tecnológica no es exclusivamente seguimiento informativo sino que se trata de un procedimiento mucho más complejo que abarca el proceso informativo-documental completo, rematado con una adecuada preparación y presentación de toda esa información de inte-

rés para la generación de inteligencia y la consecuente toma de decisiones con garantías de éxito.

En este sentido, es importante destacar los "servicios de inteligencia" que van más allá del trabajo documental, ya que implican no sólo la localización de información por parte de un grupo de profesionales sobre un sector de actividad en concreto, sino también su lectura, análisis y la correspondiente extracción de conclusiones y orientaciones relevantes y prácticas para las empresas que son reflejadas en un documento de síntesis o un informe para las mismas. La denominación "servicios de inteligencia" no es casual y viene a establecer un vínculo entre gestión de información y la inteligencia competitiva. Son destacables los puestos en marcha por compañías como Baquía o Infonomía. El de Baquía, empresa de la Red dedicada la nueva economía, elabora informes con

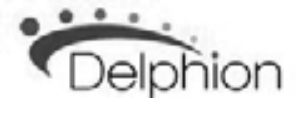

aspectos clave de interés para la gestión de empresas en internet y las nuevas tecnologías. Por su parte, a través de la segunda opción se ofrece un servicio de elaboración de informes sobre el estado de la cuestión en un área determinada del conocimiento, especialmente sobre el impacto de la tecnología en ese campo.

http://www.baquia.es

http://www.infonomia.com

\section{Familia de conceptos}

Alrededor de vigilancia tecnológica e inteligencia competitiva existen otros términos asociados, a veces poco diferenciados, que se integran todos ellos en el campo de la innovación. Una de las expresiones más generales sería la gestión de la innovación, concepto

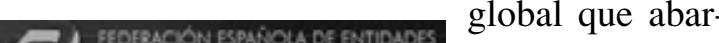
ca a todos los demás, que persigue el establecimiento de un sistema de renovación y que la llevan a cabo sólo las grandes empresas o la $A d$ ministración, pues se trata de una operación a gran escala que contempla la puesta en marcha de muchos procesos: gestión de recursos tecnológicos, tareas de vigilancia tecnológica, financiación de la innovación, transferencia de tecnología, etc.

\section{«La vigilancia tecnológica no es exclusivamente seguimiento in- formativo sino que abarca el proceso informativo-documen- tal completo, rematado con una adecuada preparación y presen- tación de toda esa información»}

La vigilancia tecnológica y la inteligencia competitiva son actividades que forman parte de un sistema de innovación y que se diferencian del espionaje industrial porque no sobrepasan los límites legales y éticos. También dentro de los límites legales está el benchmarking, técnica que consiste en la observación y análisis de procesos y productos de la competencia con el objetivo de compararlos con los propios e introducir así mejoras en los mismos.

La prospectiva tecnológica, por su parte, tiene muchos puntos en común con la vigilancia por cuanto observa y analiza el entorno para saber qué está sucediendo en un determinado sector, pero se distingue por tratar de anticiparse al futuro, de prever qué es lo que puede ocurrir teniendo en cuenta lo que sucede en el momento del análisis. Al hilo de todas estas actividades, la gestión del conocimiento se presenta como una 
herramienta de apoyo necesaria para el correcto funcionamiento de todas ellas.

\section{La información y las fuentes para la vigilancia tecnológica}

La disposición de las empresas para competir, innovar y avanzar está directamente relacionada con la capacidad para captar, procesar y entender las señales informativas de todo tipo que genera ese entorno. Aunque generalmente se habla de vigilancia tecnológica porque es la tecnología la que finalmente determina la habilidad innovadora de una compañía, la que pone en marcha una organización, se refiere también a aspectos del contexto que inciden de una u otra forma en el desarrollo de su actividad. Por ello, la información con la que deben tratar es de distinto tipo, desde la más genérica (referida a la situación social, económica, política, legislativa, etc.), hasta la más específica, es decir, aquella que tiene que ver con el desarrollo del sector de actividad que le es propio. De ahí, la variedad de fuentes de información que debe manejar.

\section{«La vigilancia tecnológica y la inteligencia competitiva se dife- rencian del espionaje industrial porque no sobrepasan los lími- tes legales y éticos»}

La relación que se muestra a continuación agrupa las fuentes y los tipos de información que más pueden aportar a las organizaciones que pongan en marcha un sistema de vigilancia tecnológica:

- Bases de datos generales.

—Bases de datos especializadas.

-Prensa general nacional e internacional.

-Prensa especializada nacional e internacional.

-Publicaciones científicas.

- Información recogida en la asistencia a ferias, congresos, etc.

-Información de clientes.

—Información de las empresas de la competencia.

-Información sobre proveedores.

-Información legislativa.

-Información estadística.

-Información normativa.

-Información no formal (conocimiento tácito): no recogida en documentos, rumores, "know-how", conocimiento de los procedimientos, etc.

Especialmente interesantes son, además, las patentes. A través de ellas se puede obtener información

tecnológica puntera que puede ser muy interesante para las empresas. El análisis de bases de datos de patentes orientado a la vigilancia tecnológica permite conocer qué temas se están investigando y quiénes lo están haciendo, detectando así nuevas tendencias y nuevas áreas abarcadas por la competencia. Para la obtención de estos resultados se han utilizado los mapas tecnológicos. Algunas fuentes básicas sobre este tipo de documentos son: la Oficina Europea de Patentes, la Oficina Española de Patentes y Marcas (Oepm), la Oficina Norteamericana de Patentes y Marcas y la base de datos de patentes de $I B M$.

http://www.european-patent-office.org

http://www.oepm.es

http://www.uspto.gov

http://www.delphion.com/

\section{Agentes en vigilancia tecnológica y áreas afines}

Aunque la actividad profesional de los gestores de información en vigilancia tecnológica e inteligencia competitiva en el contexto español es escasa (por desconocida o incipiente), o precisamente por ello, conviene conocer cuáles son los principales actores en este campo y qué se está haciendo, con el objetivo no sólo de introducirse en la materia sino también de detectar posibles áreas de actuación.

\section{«El servicio 'spidering' de DOC6 permite analizar periódicamente algunas direcciones web que pueden ofrecer datos de interés para el cliente»}

En este apartado se han querido reflejar y describir algunos de estos participantes en España, así como algunos portales de interés para el área. En esta ocasión sólo se han incorporado dos recursos extranjeros, además de los portales: la Society of Competitive Intelligence Professionals (Scip), como representante de las asociaciones profesionales, ya que no existe ninguna otra a nivel nacional, y el CERAM francés.

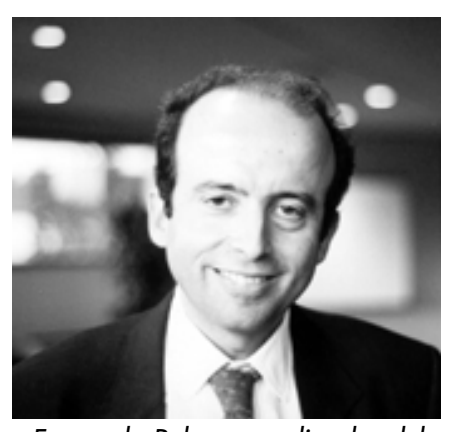

Fernando Palop, coordinador del Capítulo Español de la Scip y director de Triz XXI SL a. Empresas con una dedicación casi en exclusiva a la vigilancia tecnológica o a la tecnología en general.

\section{- Iale Tecnología.}

Segregada de la Universidad Politécnica de Cataluña, pionera en España junto a Triz XXI en vigilancia tecnológica, 
ofrece servicios de distinto tipo a otras empresas con afán innovador para la resolución de sus problemas tecnológicos. Merecen especial atención los productos relacionados con el análisis bibliométrico como la realización de mapas tecnológicos o de estudios de producción científica y otros más generales como la evaluación de sistemas de gestión de conocimiento de las empresas clientes. Iale Tecnología cita entre sus áreas de actuación las siguientes: análisis, estudios, comparaciones de parques tecnológicos, auditorías tecnológicas, formación en gestión de la innovación y tecnología, proyectos de vigilancia tecnológica, evaluación de sistemas de gestión del conocimiento y análisis de producción científica.

http://www.iale.es

\section{-Triz XXI.}

Empresa del programa Ideas de la Universidad Politécnica de Valencia, creada por José Miguel Vicente Gomila y Fernando Palop, que toma su nombre de un método utilizado para resolver procesos y crear productos mediante soluciones innovadoras. La aplicación de este procedimiento permite no sólo analizar los problemas generados en las organizaciones sino también prever qué puede ocurrir en un futuro próximo, acercándose así al campo de la prospectiva.

\section{«Baquía elabora informes con aspectos clave de interés para la gestión de empresas en inter- net y las nuevas tecnologías»}

Su principal misión es favorecer la innovación en las empresas cliente apoyándose para ello en los procesos de vigilancia tecnológica, en la gestión del conocimiento y en la creatividad. Además, parten de la base de que existen conocimientos y tecnologías en determinados sectores que pueden ser aplicados en otros por lo que observan entornos distintos al que analizan en cada momento, haciendo de la interdisciplinariedad una forma de trabajo.

Entre otras cosas, Triz XXI ha diseñado un "puzzle tecnológico", herramienta que aporta información a los empresarios sobre el estado de la vigilancia tecnológica en su organización. Además es la distribuidora oficial en España de un programa informático que facilita la innovación para las empresas a partir de la gestión de distintos tipos de conocimientos. Sus páginas web son una referencia obligada en este campo en España y dan acceso, entre otras cosas, a Triz journal en español, selección de artículos de la revista original Triz journal, acompañada de sus correspondientes comentarios.

http://www.triz.net

http://www.triz.es

\section{-Robotiker.}

Es una fundación con sede en el País Vasco dedicada a la innovación tecnológica y que suministra servicios a sus clientes sobre las diferentes fases del proceso innovador: desde la vigilancia tecnológica hasta el desarrollo de proyectos de I+D pasando por actividades tanto de consultoría como de formación y el apoyo a la creación de nuevas empresas de base tecnológica. Aunque está especializada en tecnologías de la información, trabaja también en otras áreas como la electrónica, telecomunicaciones, ingeniería industrial, etc.

\section{«El benchmarking consiste en la observación y análisis de pro- cesos y productos de la compe- tencia con el objetivo de compa- rarlos con los propios e introdu- cir así mejoras en los mismos»}

Ofrece dos servicios informativos gratuitos relacionados con las tecnologías emergentes: Eraiki@news es un boletín de vigilancia tecnológica según la denominación de la propia empresa, que informa cada quince días de las últimas novedades; y Re-vista, publicación electrónica que además acoge una sección sobre gestión del conocimiento.

\section{http://www.robotiker.com}

b. Empresas de documentación con servicios o productos de vigilancia tecnológica. En este grupo se pueden diferenciar las que desarrollan todo tipo de ta-

El profesional de la información está abierto a todos los bibliotecarios, documentalistas y profesionales de la información, así como a las empresas y organizaciones del sector para que puedan exponer sus noticias, productos, servicios, experiencias y opiniones.

Dirigir todas las colaboraciones para publicar a:

\section{El profesional de la información}

Apartado 32.280

08080 Barcelona

Fax: +34-934250 029

epi@sarenet.es 
reas documentales $y$ que han empezado a ofrecer ser-

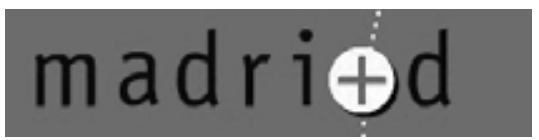
vicios específicos

(como el "spidering" mencionado anteriormente) y a participar en actividades de vigilancia tecnológica en empresas (Odei, DOC6, etc.); y las que están dedicadas casi en exclusiva al seguimiento sistemático de fuentes para analizar y difundir entre sus clientes las informaciones que respondan a sus necesidades, como es el caso de MyNews Online o, la más orientada a la vigilancia, CDEtracker \& Buzzcity.

http://www.odei.es

http://www.mynewsonline.com

http://www.cde.es

c. Empresas de otros sectores que cuentan con sus propios servicios de documentación y vigilancia tecnológica. Es el caso del Instituto Tecnológico Agroalimentario, el centro tecnológico Gaiker, especializado en plásticos, medio ambiente y biotecnología industrial o el Instituto Tecnológico del Plástico (Aimplas), por citar sólo algunos ejemplos.

http://www.ainia.es/bases/vt/vt.nsf

http://www.gaiker.es/Pages/docu.htm

http://www.aimplas.es/servicios/documentacion/oferta servicios.html

d. Instituciones de carácter público.

-Observatorio de Prospectiva Tecnológica e Industrial (Opti).

Es una fundación cuyo objetivo es el estudio del impacto de las tecnologías en los distintos sectores de actividad (oportunidades, riesgos, tendencias, procesos de adopción de tecnologías, etc.) a largo plazo. Por ello, pretende ayudar en la toma de decisiones tanto a las empresas como a las administraciones. Periódicamente elabora estudios de prospectiva para distintos sectores y publica el Boletín Opti mediante el cual se presentan informaciones de prospectiva tect $\mathbf{r}$ i $\mathbf{z}_{X \times 1}$ nológica. Aunque ésta es su principal área de actividad, otro de sus campos de trabajo es la vigilancia.

http://www.opti.org (Ipts).

_Instituto de Prospectiva Tecnológica de Sevilla

Forma parte, junto con ocho institutos más, del Joint Research Centre (JCR) de la Comisión Europea.
La función de todos ellos está encaminada al análisis de la ciencia y la tecnología, sobre todo lo que concierne a temas emergentes, con el objetivo de proporcionar directrices útiles a aquellas personas encargadas de la toma de decisiones. La conexión de los tres polos: análisis económico, ciencia y tecnología y política, es una de sus actuaciones más importantes, así como uno de los modelos que se están tratando de potenciar cuando se habla de investigación y desarrollo.

El propio nombre del centro indica su vinculación con la prospectiva y, en cierto modo,

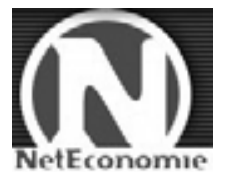
con la vigilancia tecnológica. Analiza la situación del entorno y trata de prever en qué forma puede afectar a los distintos agentes de un sistema y a la política que se desarrolle en torno al tema.

El Ipts elabora periódicamente informes técnicos y edita la revista mensual The Ipts report, especializada en ciencia y tecnología, política científica y desarrollo económico, fundamentalmente referidos al ámbito europeo.

http://www.jrc.es/

http://www.jrc.es/pages/f-report.en.html

— Ministerio de Ciencia y Tecnología.

Ofrece un servicio de vigilancia tecnológica a través de boletines trimestrales de información, realizados por la Oficina Española de Patentes y Marcas y por el Observatorio de Prospectiva Tecnológica Industrial. Esta publicación suministra información de carácter tecnológico y empresarial, incluyendo nuevas

\section{e-robotiker} solicitudes de patentes, para el área agroalimentaria y para sectores bási-

cos y transformadores.

http://www.mcyt.es

—Ingenio. Instituto de Gestión de la Innovación y del Conocimiento.

Es un centro mixto de la Universidad Politécnica de Valencia y el Consejo Superior de Investigaciones Científicas, dedicado a la investigación y a actividades de formación y consultoría en innovación y gestión del conocimiento, entendiendo éste como una de las bases de la innovación.

http://www.ingenio.upv.es/

\section{—Instituto Catalán de Tecnología.}

Es una fundación dedicada a presentar servicios a empresas para mejorar su capacidad innovadora, des-

Veille.cQm de el punto de vista tecnológico, ofreciendo servicios de forma- 
ción, información, desarrollo de proyectos de I+D y consultoría. Una de sus prestaciones más interesantes es el seguimiento de la información tecnológica para suministrarla a las compañías, realizando así parte de sus actividades de vigilancia.

ICTnet mantiene y da acceso a distintas comunidades virtuales entre las que se encuentra una dedicada a gestión de la innovación. A través de sus páginas se puede acceder a informaciones de distinto tipo, documentos a texto completo y a una lista de discusión especializada. Destaca la recopilación de recursos comentados sobre innovación en el apartado "centros de apoyo a la innovación".

http://www.ictnet.es

$-M a d r i+d$.

La vigilancia tecnológica, la inteligencia competitiva y la gestión del conocimiento son herramientas de trabajo presentes en el Plan regional de investigación científica e innovación tecnológica (2000-2003) de la Comunidad de Madrid. Cabe destacar, entre otras cosas, el proyecto de los círculos de innovación incluido dentro del Sistema Regional de Información y Promoción Tecnológica Madri+d. Los círculos de innovación representan una forma organizada de prestar servicios de vigilancia tecnológica a las empresas madrileñas incluidas en un sector determinado de acuerdo con las necesidades de cada una de ellas. Hasta el momento son cuatro las áreas activas: biotecnología, materiales, medio ambiente y energía y tecnologías de la información y las comunicaciones.

\section{http://www.madrimasd.org}

Otros servicios de información regionales relacionados con la innovación y la tecnología son la Red Vasca de Tecnología, el Centro de Innovación y Servicios de Galicia, el Centro Europeo de Empresas e Innovación de Navarra o la Red Andalucía Innovación.

http://www1.euskadi.net/saretek_erakus/setrvt_c.asp

http://www.xunta.es/auto/index.htm

http://www.cein.es/

http://www.iat.es/red/

e. Asociaciones profesionales.

- Society of Competitive Intelligence Professionals (Scip).

Es la asociación internacional más relevante para los profesionales de la inteligencia competitiva. Fue creada en 1986 y tiene su sede principal en Virginia (EUA), pero cuenta con numerosos capítulos o delegaciones en distintos países del mundo. En España fue presentada a principios del año 2000, y es el profesor Fernando Palop, de la empresa Triz XXI, su contacto español. Cuenta con unos 7.000 afiliados y un alto porcentaje de los mismos (77\%) proceden del mundo empresarial.

Entre las distintas actividades que desempeña en relación con la inteligencia competitiva están las de formación, intercambio de información entre profesionales y divulgación de este campo del conocimiento, definido por la propia asociación como interdisciplinar pero situado fundamentalmente entre dos áreas: la gestión de la información y la gestión empresarial. El gestor del conocimiento es el profesional protagonista en este proceso.

\section{«ICTnet mantiene y da acceso a distintas comunidades virtuales entre las que se encuentra una dedicada a gestión de la innova- ción»}

En las páginas institucionales se puede encontrar una amplia información sobre la asociación, sus actividades y la inteligencia competitiva en general. Además, edita dos publicaciones relacionadas con este tema:

-Scip's CI magazine, revista profesional de carácter trimestral en la que publican especialistas en inteligencia competitiva. A través de sus páginas se ofrece una selección de los artículos más recientes a texto completo.

-Scip's CI review journal, de naturaleza científica que ve la luz cuatro veces al año.

http://www.scip.org

f. Selección de portales y guías de recursos especializados en vigilancia tecnológica e inteligencia competitiva.

—Veille.com.

Se puso a disposición del público a partir de la edición del libro de Carlo Revelli, Intelligence stratégique sur internet (Dunod, $2^{\mathrm{a}}$ edición, 2000), como una prolongación derivada de la publicación impresa. Ha sido una iniciativa de la empresa Cybion (On line Business Intelligence), con la idea de agrupar en una sola sede web las diferentes experiencias en inteligencia estratégica comunicadas en especial a través de foros de discusión y listas.

A través de sus páginas se ofrecen los siguientes servicios:

- Cursos tanto de formación en vigilancia tecnológica como orientados al manejo de las herramientas web para la búsqueda de información.

- Servicios de alerta para la gestión de situaciones de crisis en las empresas. 


\title{
Próximos números monográficos
}

\author{
Septiembre 200I Archivos digitales en empresas y organizaciones \\ Diciembre 200I Intranet y documentación
}

Marzo 2002 Automatización de bibliotecas

Los interesados pueden remitir notas, artículos, propuestas, publicidad, comentarios, etc., sobre estos temas a:

epi@sarenet.es

—Realización de estudios sectoriales.

-Unidades de vigilancia económica y tecnológica en internet a partir de agentes inteligentes.

-Búsquedas puntuales de información en la Red.

-Análisis de los sitios web referenciados en los motores de búsqueda.

Además, pone a disposición de los usuarios algunos recursos gratuitos:

-Un laboratorio de agentes inteligentes (de búsqueda y de vigilancia).

-Fichas técnicas de los principales agentes inteligentes de búsqueda y vigilancia tecnológica. tema.

-Acceso a una comunidad virtual especializada el

-Utilización de los archivos de todas las listas de discusión sobre este campo de actuación.

—Comunidad virtual sobre agentes inteligentes.

-Acceso libre a un anuario de agentes inteligentes.

-Acceso libre al Anuario de inteligencia económica y de knowledge management.

-Ofrece un pequeño manual titulado La vigilancia tecnológica en 10 lecciones para formación básica.

http://www.veille.com/

—Neteconomie.fr: l'Economie du net.

NetEconomie.fr es una publicación electrónica de NetEconomie Sarl, que suministra una serie de recur- sos todos ellos relativos a la economía en la Red. Señalamos los más interesantes:

- Servicio de alerta sobre noticias de actualidad de nuevos productos industriales, comercio electrónico, noticias de empresas e informaciones sobre fenómenos sociales en relación con internet.

- Informes específicos sobre la economía en la Red de acceso gratuito para los siguientes grandes temas: productos industriales, comercio electrónico, vigilancia y gestión del conocimiento.

- Guía y acceso a los principales clubs de economía en la Red.

- Sumario de servicios de los actores de la economía en la Red: comercio electrónico, agencias, inversiones, formación, operadores, servicios en línea, etc.

http://www.neteconomie.com

\section{-Brint.com.}

Es el primer portal sobre gestión del conocimiento y la comunidad virtual global para la nueva economía mundial. Está creado por la empresa@Brint.com LLC y ofrece en sus páginas acceso a múltiples recursos especialmente fuentes de información, documentos a texto completo, noticias de actualidad y una amplísima comunidad virtual especializada en gestión del conocimiento que se expresa a través de listas y foros de discusión. En este marco, la "business intelligence" es una parte de la gestión del conocimiento y como tal está cubierta en los diferentes servicios y recursos que oferta este portal.

http://km.brint.com 


\section{Versión online de EPI}

Existe una versión electrónica de la revista El profesional de la información, de uso gratuito para la mayoría de los suscriptores (empresas, organismos, instituciones), consultable en:

http://www.swetsnet.nl/

Más información en:

http://www.szp.swets.nl/szp/journals/pi.htm

Además, en el ámbito español, es necesario mencionar la comunidad virtual de gestión de la innovación del Instituto Catalán de Tecnología (véase apartado d), la recopilación de recursos comentados sobre inteligencia competitiva incluida en el portal Infonomía, así como las pequeñas guías de recursos incorporadas a algunas de las fuentes comentadas (Triz XXI, Ma$d r i+d$, etc.)

http://www.infonomia.com/suma/index.asp?tema $=2$

g. Otras fuentes.

-Cotec.

La Fundación Cotec para la Innovación Tecnológi$c a$ ha promovido la realización y publicación de estudios sobre vigilancia tecnológica, entendiendo que ésta es una herramienta importante para el desarrollo de las empresas en un entorno competitivo. Todos estos análisis permiten observar la relación de los procesos de vigilancia tecnológica con los sistemas de gestión de la información y los procedimiento de gestión del conocimiento. Además, su línea de trabajo (indicadores de ciencia y tecnología y, por tanto, en contacto con el análisis bibliométrico) contribuye a la aproximación de los campos temáticos información y empresa.

http://www.cotec.es

-Federación Española de Entidades de Innovación y Tecnología (Fedit).

Sus miembros son centros tecnológicos encargados de poner en contacto los resultados de la investigación con las necesidades de la empresa. Para ello ofrecen distintos servicios, entre los que se encuentran la difusión tecnológica y la información, facilitando así las funciones de vigilancia tecnológica que, a mayor o menor escala, puedan desarrollar las pymes.

http://www.fedit.es
-Ceram Sophia Antipolis. Graduate School of Management \& Technology.

Es un centro universitario especializado integrado en el complejo de Sophia Antipolis, en las inmediaciones de Niza (Francia) y tiene a gala ser el pionero en Europa en impartir un máster especializado en inteligencia económica y gestión de proyectos complejos. Se presenta con el lema "gestionar la incertidumbre y la complejidad: una nueva profesión". Sus páginas contienen amplia información sobre este y otros programas de máster y doctorado. En este artículo transcribimos, por su interés para los profesionales de la información, el programa del máster que ofrece en el campo que nos ocupa.

http://www.ceram.edu/V2/

\section{Nota}

Estudio realizado en el marco del programa de becas de formación de personal investigador de la Dirección General de Investigación de la Comunidad Autónoma de Madrid (CAM).

\section{Bibliografía}

Adbs. Commission Métiers et Qualifications. Métiers de l'information et documentation. Consultado en: 20-04-2001.

http://www.adbs.fr/adbs/viepro/profess/metier/html/1n3vpm22.htm

Euroreferentiel I\&D. Référencial des compétences des professionnels européens de l' information et documentation. Paris: Adbs Éditions, 1999, p. 27. Isbn 2-84365-027-5.

Giménez Toledo, Elea. "Campo de acción de la documentación en actividades de vigilancia tecnológica”. En: VII Jornadas españolas de documentación: la gestión del conocimiento: retos y soluciones de los profesionales de la información, 2000, pp. 507-515.

Maspons, Ramón. "Inteligencia económica y vigilancia tecnológica". (documento de trabajo). En: Seminario de los círculos de la innovación, 1999.

Palop, Fernando; Vicente, José Miguel. Vigilancia tecnológica e inteligencia competitiva. Su potencial para la empresa española. Madrid: Cotec, 1999.

Palop, Fernando; Vicente, José Miguel. Vigilancia tecnológica. Madrid: Cotec, 1999.

¿Qué es la vigilancia tecnológica? Madri+d. Consultado en: 09-04-2001. http://www.madrimasd.org/servicios/circulosinnovacion/ie-vt.asp

Relación de eurocompetencias en información y documentación. Madrid: Sedic, 2000 